\title{
Gaze-based teleprosthetic enables intuitive continuous control of complex robot arm use: writing $\&$ drawing
}

\author{
Sabine Dziemian ${ }^{1}$, William W. Abbott ${ }^{2}$, and A. Aldo Faisal ${ }^{1,2,3}$, Member IEEE
}

\begin{abstract}
Eye tracking is a powerful mean for assistive technologies for people with movement disorders, paralysis and amputees. We present a highly intuitive eye tracking-controlled robot arm operating in 3-dimensional space based on the user's gaze target point that enables tele-writing and drawing. The usability and intuitive usage was assessed by a "tele"writing experiment with 8 subjects that learned to operate the system within minutes of first time use. These subjects were naive to the system and the task and had to write three letters on a white board with a white board pen attached to the robot arm's endpoint. The instructions are to imagine they were writing text with the pen and look where the pen would be going, they had to write the letters as fast and as accurate as possible, given a letter size template. Subjects were able to perform the task with facility and accuracy, and movements of the arm did not interfere with subjects ability to control their visual attention so as to enable smooth writing. On the basis of five consecutive trials there was a significant decrease in the total time used and the total number of commands sent to move the robot arm from the first to the second trial but no further improvement thereafter, suggesting that within writing 6 letters subjects had mastered the ability to control the system. Our work demonstrates that eye tracking is a powerful means to control robot arms in closed-loop and real-time, outperforming other invasive and non-invasive approaches to Brain-MachineInterfaces in terms of calibration time ( $<2$ minutes), training time ( $<10$ minutes), interface technology costs. We suggests that gaze-based decoding of action intention may well become one of the most efficient ways to interface with robotic actuators - i.e. Brain-Robot-Interfaces - and become useful beyond paralysed and amputee users also for the general teleoperation of robotic and exoskeleton in human augmentation.
\end{abstract}

\section{INTRODUCTION}

We observe the world through discrete, rapid, focussed eye movements (saccades) acting to align the high-resolution central vision area (fovea) of both eyes with an object of interest (fixation point). Visual information is vital to motor planning and thus monitoring eye movements gives significant insight into our motor intentions. Thus by understanding the perception-action-loop we can interpret eyemovements to provide a high-bandwith, low-latency signal directly relevant for neuroprosthetic control. Our aim is to harness 3D eye movements for assistive robotics. The goal of our system is to produce a robotic platform that is comparable with an assisting human, rather than the limited functionality platforms typical of prosthetics. By moving the prosthesis off the body we eliminate many issues relating to human interaction safety, human physical strength and endurance, weight and loading capacity, battery life, and

Brain \& Behaviour Lab - ${ }^{1}$ Dept. of Computing \& ${ }^{2}$ Dept. of Bioengineering, Imperial College London, SW7 2AZ London, ${ }^{3}$ MRC Clinical Sciences Centre, Hammersmith Hospital, London, UK. Address for correspondence: aldo.faisal@imperial.ac.uk control interface. Separating the prosthetic from the user by making it a "teleprosthesis" is a key insight that will allow us in the future to construct useful assistive technology using state-of-the-art dexterous robotics.

Intuitive end-point control of the end-effector of a robot arm is the necessary component for any dexterous manipulation system - our goal is that the system should be ultimately capable of performing all the helper tasks that a human would perform for the user, and our goal is that system will allow the user to perform these tasks themselves, quickly and efficiently [1], [2], [3]. Recently, 3D eye tracking emerged as a very powerful interface for paralysed and amputees to interact with their environment: It is particularly suitable as many causes of partial or full paralysis do not affect or only little the patient's ability to move the eyes in a goal-

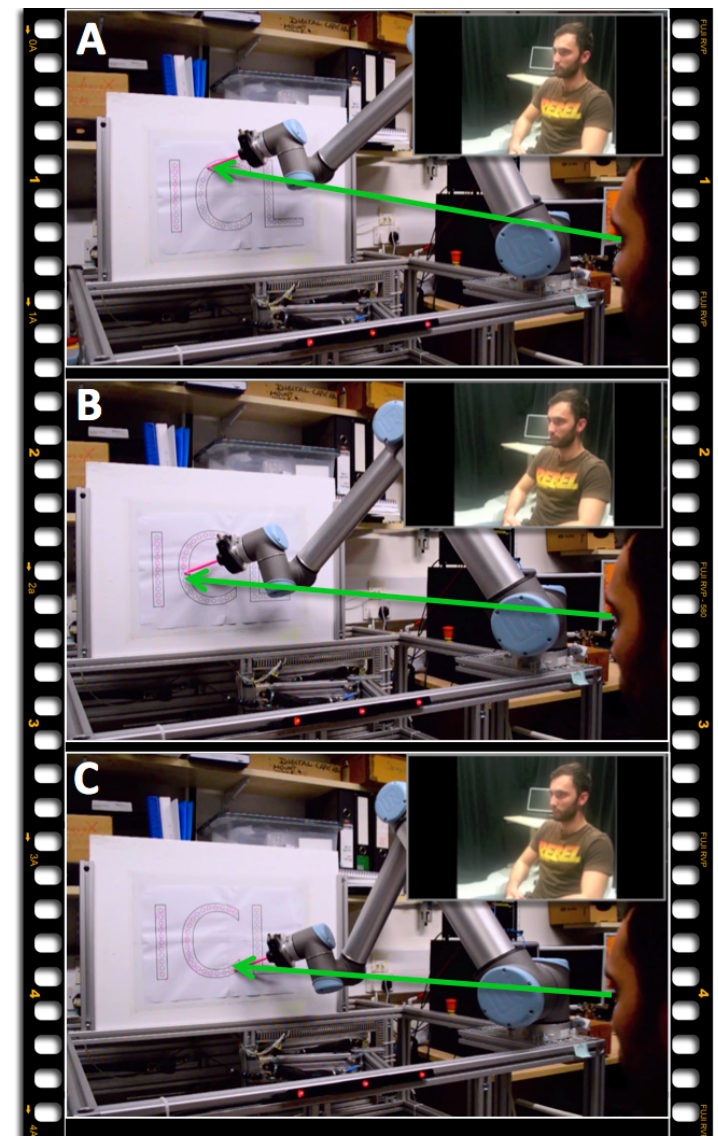

Fig. 1. Subject controlling the robot arm with a closed-loop interaction scheme. To the left is the canvas, to the right the chair for the user, looking to the front of the rectangular workspace with eye tracking bar and to the robot. (A-C)The robot arm moves exactly to the point at which the subject is fixating with the eyes. The arm writes whenever the tip touches the canvas. 
directed manner [1], [4]. For all people living with some form of paralysis performing daily-life activities is substantially hindered or even impossible. Developing a gaze-controlled robot arm attempts to make those people's lives easier by restoring vital degrees of freedom and restores basic armtype functionality.

Gaze-based assistive were used to type and draw on screen-based computer application before, e.g. [5] . We use eye tracking to control the end-point of the robot in a continuous fashion. Moreover, the fact that natural eye movements are highly action intention-related and particularly suitable for decoding physical interaction intentions[6]. Gaze-based action selection have been used before for robot action selection, but not continuous end-point control. Electrical eye signals (EOG) were used to detect blinks and these blinks then triggered a humanoid robot to trigger a grasp, whereby the number of blinks determined which hand of the robot was actuated [7]. The challenge lies in blinks being naturally occurring events, essential for eye health, and the end-point control of the robot required joystick manipulation. In more recent application 2D gaze targets were used to select motion sequences that were triggered by gazing at the appropriate panel on the screen[2]: The user has to interact with a screen based interface which enables decoding of target endpoint and is combined into a control system that boosts the intended trajectory estimate obtained from (considerably more noise) EMG arm muscle recordings and guides the robotic arm. In contrast, we demonstrate free $3 \mathrm{D}$ end-point control capabilities. The focus is on the integrated operation of the 3D end-point of the robot effector for rather complex tasks such as writing and drawing based on gaze targets. We specifically do not address here a more general solution to the Midas Touch problem, i.e. the challenge of distinguishing between different action intentions at a gaze end point or distinguishing action related from non-action related visual attention, and simply use basic dwell times to filter these out.

We previously deployed our free-gaze control approach in a number of continuous control scenarios. We conducted a large-scale experiment by putting up eye tracking "booths" to enable users to play the arcade game pong at science fairs. This resulted in field-trial with hundreds of naive users, which after un-assisted calibration enabled over $88 \%$ of users were able to play successfully the arcade video game Pong in under 60 seconds (measured from stepping up to the device, automatic calibration, and scoring in Pong) [4] - thereby demonstrating the gaze-based continuous control is effective and can be learned very quickly. We enabled the free-gaze cursor approach by developing natural gazebased controllers for wheelchairs[3]: Volunteers drove a wheelchair in virtual reality by operating a joystick while we simultaneously recorded their $3 \mathrm{D}$ eye movements. We then correlated the natural driving-related eye movements with the recorded driving command. This natural decoding solution was deployed on an actual electrical wheelchair and enabled users to freely drive the wheelchair without having to look at a visual display display or other user interface but just looking normally ahead of where they want to drive.
Our goal is now here to apply the free-gaze approach to continuous robot control for complex arm control tasks, namely drawing and writing with a pen on canvas. In this paper, eye movements are used as a 3D end-point cursor in the space of a canvas for writing and head tilts are used to control the robot's motion in the third dimension. The Midas Touch problem in intention decoding from eye tracking results from the ambiguity of task-related and non-unrelated eye movements being confounded[8], [1]. To resolve this problem, we use here a simple combination of wink and dwell time that recognises selective eye movements related to pen use. We assess the intuitiveness of this approach, by having subjects physically writing three capital letters on a canvas with a felt tip pen attached to the endpoint of the robot arm. The total time the subjects needed to complete the task (from first time use) and the total number of selective commands sent was used to indicate the level of confidence the subject gained during use.

\section{SyStem OVERVIEW}

Our goal is to develop a tele-prosthetic control system in which a user can intuitively and continuously control the movement of a robot arm with their eyes to physically write or draw with a pen attached to the robot arm's end-point. The system consists of the low-cost Tobii EyeX Controller (Tobii Technology, Danderyd, Sweden) and the UR10 robot arm (Universal Robots, Odense, Sweden).

a) Devices: The Tobii Eye X Controller is a remote eye tracking bar normally used for screen-based interactive applications . It reports the two-dimensional gaze and fixations points of the eyes on a computer screen. The system also returns head tracking information in form of the position of both eye balls within the head track box. Effective and reliable usage is supported for physical visual display units with a maximum size of 27 inch (manufacturer specification). The optimal distance from the user to the eye tracker lies between $60 \mathrm{~cm}$ and $80 \mathrm{~cm}$. The framerate is approx. $50 \mathrm{~Hz}$ and the system uses different error codes if the data is not considered valid because of closed or not detected eyes. The system is delivered with a calibration routine for a $2 \mathrm{D}$ computer screen that is built-in to the driver. We chose this consumer-entry level device, effectively a computer game gadget, to explore the capability for gaze-based controlled using mass-marketed low-cost devices $(<200 \$)$ universally obtainable. Significantly better performance can be straightforward achieved with head-mounted research-grade devices that can perform true 3D eye tracking, such as GT3D [1]. Moreover, a 3D eye tracker can provide fully free 3D control of the robot arm. In future work we will be also exploring the use of head-worn eye-trackers with actuated prosthetic arms.

The UR10 is a 6-axis-arm robot with 6 degrees of freedom. It can operate in a working radius of $1.3 \mathrm{~m}$ and allows to reach for any point within that radius, apart from the space occupied by the robot's base. It provides a joint range of $\pm 360^{\circ}$. The maximal payload of the arm is $10 \mathrm{~kg}$ and is sufficient to enable future mounting of dexterous robotic 
hand actuators. A felt-tip pen is mounted perpendicular to the end-point surface of the robot. The loop for processing user control commands operates at approx. $100 \mathrm{~Hz}$. While the robot system comes at considerable cost (factor 200 times the cost of the eyetracker) it provides a stable platform to test eye-based control of robotic systems - as the focus of the work is on if and how gaze-tracking can be used to control robots, less on the actual robotic system deployed.

The computer processing the inputs and running the control commands was operating with a loop delay of 10 milliseconds or less, enabling processing of eye tracking and arm control with only small aliasing effects in timing.

b) Setup: The user is sitting in front of the rectangular work space (see Figure 2.A). The robot arm is mounted on the right corner of the table - to the right from the user's perspective for right-handed users. On the opposite side of the user in a distance of approximately $87 \mathrm{~cm}$ a white plane of the size of of $84 \times 59 \mathrm{~cm}$ is attached to the frame, which acts as mounting surface for the canvas. This plane shows nine points which match the calibration points used by the Tobii Engine for screen based calibration. Although the calibration points are at a greater distance they correspond to positing a 27 inch visual display unit at approximately $70 \mathrm{~cm}$ to the user. This enlargement of the interaction region of the work space is effective, because the eye tracker's reconstruction of the both eye vectors remains consistent independent of canvas distance.

The eye tracking bar is attached to the front edge of the table and at an angle of $20^{\circ}$. The user is sitting in a distance of approximately $70 \mathrm{~cm}$ from the table with the eye tracker. To the eye-tracker it appears that the eyes are tracked as if the user was looking at a 27 inch visual display unit standing atop the eye tracker. In reality there is no computer screen, and the user is looking straight at the canvas from a total distance of approximately $157 \mathrm{~cm}$ enlarges the interaction and control workspace for the robot arm. With the drawing plane at a distance of $87 \mathrm{~cm}$ from the eye tracker a workspace of approximately $84 \times 59 \mathrm{~cm}$ has been gained. After limiting the workspace for safety cut-off features a total area of $67 \times 38 \mathrm{~cm}$ remains in the low-level robot control system. The difference between both coordinate systems needs to be taken into account when transforming gaze coordinate into robot arm workspace coordinates. Specifically this requires the combination of head- and eye-tracking coordinate systems.

c) Data Flow: The data flow of the system that closes the loop between eye-tracker, intention decoder and robot uses ROS [9] as middleware (see Figure 2.B). The gaze data is sent to the main computer with a Linux operating system. Given that the eyes are continuously trackable by the eye tracker the data is constantly transmitted in a streaming manner. The gaze data is constantly analysed for move commands to the robot which are decoded by fixations of at least $600 \mathrm{~ms}$. We use the standard fixation detection algorithm, which basically assumes that (micro)saccades within an area proportional to the fovea, are part of the same fixation. The eye tracker position stream is analysed for displacement of the eyes which indicate tilting of the head. A forward

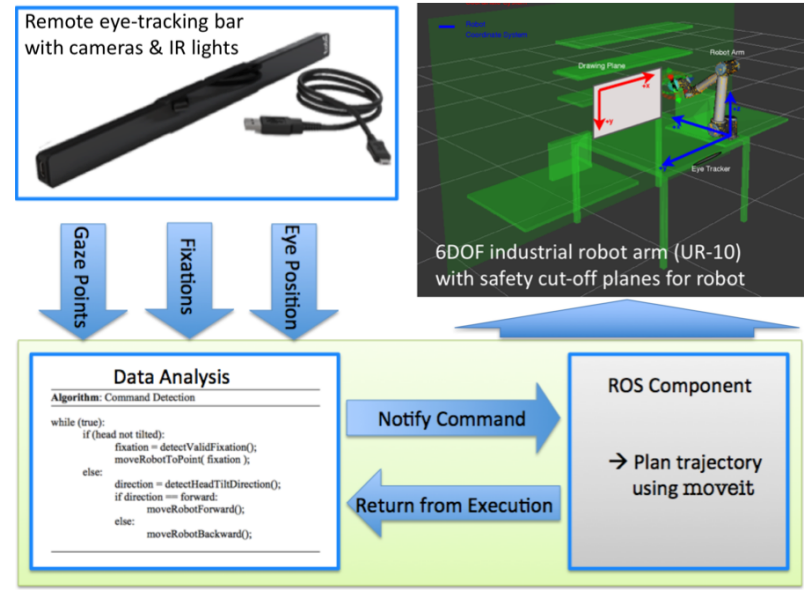

Fig. 2. Data flow diagram of device and ROS integration (see text).

tilt codes for the robot moving closer to the plane and a backward tilt for moving the robot further away from the plane, respectively. Thus, the user can decide if they want to draw/write or simply direct the pen to a different location. The commands are then processed by our ROS system on the Linux machine such that the appropriate robot motion is planned and executed using the moveit subsystem of ROS. To allow for safe motion planning the physical workspace is implemented as a collision object such that the robot will only move in non-occupied space. Furthermore, additional safety cut-off planes are inserted that separate the robot workspace from the area in which the user is sitting. As planned robot trajectories can not pass through objects it is assured no part of the robot will move into the user space. After successful robot motion the analysis for the next move commands continues.

\section{ROBOT-END POINT CONTROL BY EYE TRACKING}

In our systen we wanted to operate the robot without requiring the user interact with a visual display unit or user interface, instead we implemented a free-gaze control strategy for control. Human users have four basic types of eye movements: saccades, smooth pursuit movements, vergence movements, and vestibulo-ocular movements. Saccades are rapid, ballistic movements of the eyes that abruptly change the point of fixation. Targets of saccades can be under voluntary or unconscious control. Smooth pursuit eye movements are much slower tracking movements of the eyes designed to keep a moving stimulus in the narrow centre of high visual resolution (the fovea). Users have conscious control whether to track or not to track a moving target, but cannot generate smooth pursuit eye movements without tracking (i.e. they cannot trace a line on a static surface). Humans (and other animals with a fovea) typically alternate saccades and visual fixations. There are two more unconsciously controlled smooth eye movements: Vergence movements align the fovea of each eye with targets located at different distances from the user. Vergence movements can be used by 3D eye tracking system to detect the distance of a gaze target [1], but we do not have to rely on these due to the mostly planar drawing and writing task of the 
robot. Vestibulo-ocular movements stabilise the eyes relative to the external world by compensating for head movements, enabling the user to perform head-tilts and still maintain a fixation. For free-gaze robot end-point control we rely primarily onsaccades as these are under user control.

We calibrated the user eye positions against the canvas, removing the visual display unit completely from the system - voice commands prompted the user to move their eyes between calibration points on the canvas during the calibration phase. This enables us to use the eye trackers built in calibration routine while the user is actually looking at the white plane in a further distance (see Fig. 3.c).

The main aim of this paper is to demonstrate end-point control capability using 3D gaze controlled end-point locations. Therefore, we chose a very simple interaction scheme to decide on when eye movements indicated an intention to move the robot arm: We use dwell times to disambiguate purposeful positioning eye movements from distractor eye movements. Common dwell times in 2D screen based gazeinteraction seetings are between $500 \mathrm{~ms}$ and $1000 \mathrm{~ms}$. We chose a dwell time of $600 \mathrm{~ms}$ as it allowed a reliable flow, while accounting for the fact in 3D fixations are more variable than 2D fixations. Once the dwell time is reached we move the robot arm on a two-dimensional plane to the new gaze target. Forward and backward head tilts move the arm in the third dimension. The robot arm moves to the coordinate defined by the current gaze point after fixating at it for more than $600 \mathrm{~ms}$. Hereby, every consecutive fixation must be detected within a very small area to make it a valid selective fixation. This scheme corresponds to the position variance scheme proposed by [10]. After detecting valid fixations lasting longer than $600 \mathrm{~ms}$ the average over those points is defined as the point of selective fixation. The selective fixation point is translated into robot coordinates and sent to the UR10 Controller for the robot arm to move. During interaction acoustic feedback is provided as soon as a move command is received to lower the uncertainty of selection and to prevent the user from repeating command sending during the latency of the robot. As long as the robot is in motion no other selection commands are carried out. Commands that are sent very shortly before the end of the robot motion are executed after completing the initial motion. Hereby the user can have a rapid and smooth flow of command sending.

\section{Results: User Control Performance}

The level of intuitive usage of this application has been assessed by an experiment that consisted of the task to write the three unlinked capital letters ICL on the drawing plane using only the gaze and head tilts to control the robot arm (see Fig. 4.C) The pattern spans a size of approximately $46 \times 23 \mathrm{~cm}$. There are 50 circles within the letters. The user's goal is to draw through as many circles as possible completing all three letters within the shortest time and using as few fixations as possible. With backward and forward tilting of the head the user has to write the letters separately by attaching and detaching the pen to the drawing plane. In any experiment neither are the coordinates of the circles specified within the program nor are the letters the subject has to write known in the application. The entire control is only based on the estimate of where the subject is looking at by tracking the eyes. The experiment is repeated five times per subject to assess the performance with repetition. To allow the user to adapt to a potential offset the initial eye tracker calibration required by the Tobii Engine is only performed once at the beginning of all five trials. During use the subject is seated in a chair with armrests to minimize body movement. The following figure 4 shows the unprocessed fixation stream data provided by the eye tracker. Superimposed is the robot trajectory with the pen attached to the drawing plane in green and the pen detached in blue.

The purpose of the writing experiment was to analyse the intuitive operability of our simple interaction scheme. The experiment investigated on the level of ability and confidence in the usability the user develops from trial to trial. The number of points hit was evaluated to indicate if the system is suitable for using it for precise movements.

The total time needed to complete writing and the total number of fixations was used to give insight into the level of confidence the user develops and how fast the user can learn the control. A small number of fixations reflects a high confidence level since a straight line, i.e. the letters I and L can be drawn with only two and three fixations respectively. However, this is only achievable if the user is certain about the robots trajectory resulting from the gaze commands sent. A fast decline of the total time used would indicate intuitive operability.

The primary result that emerged from the experiment is that the number of circles hit remains constant as there was no significant difference found between trials across all subjects. This shows that subjects are indeed able to write by eye as the gaze can be controlled precisely to perform a smooth robot trajectory. Moreover, figure 4 shows that the robot movements do not interfere with the subject's natural gaze. Hence the subject is not restricted in using the gaze

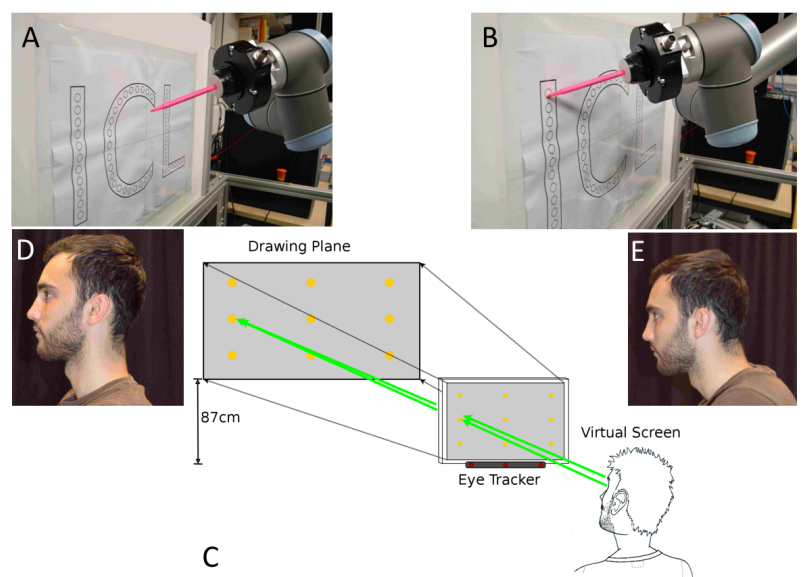

Fig. 3. The robot arm can be steered away or towards the canvas so that the pen is contact with the canvas or away from it $(A, B)$ by head tilts $(D, E)$. (C) During calibration the virtual screen (not existent) is at the same distance as the remote eye tracking bar. 

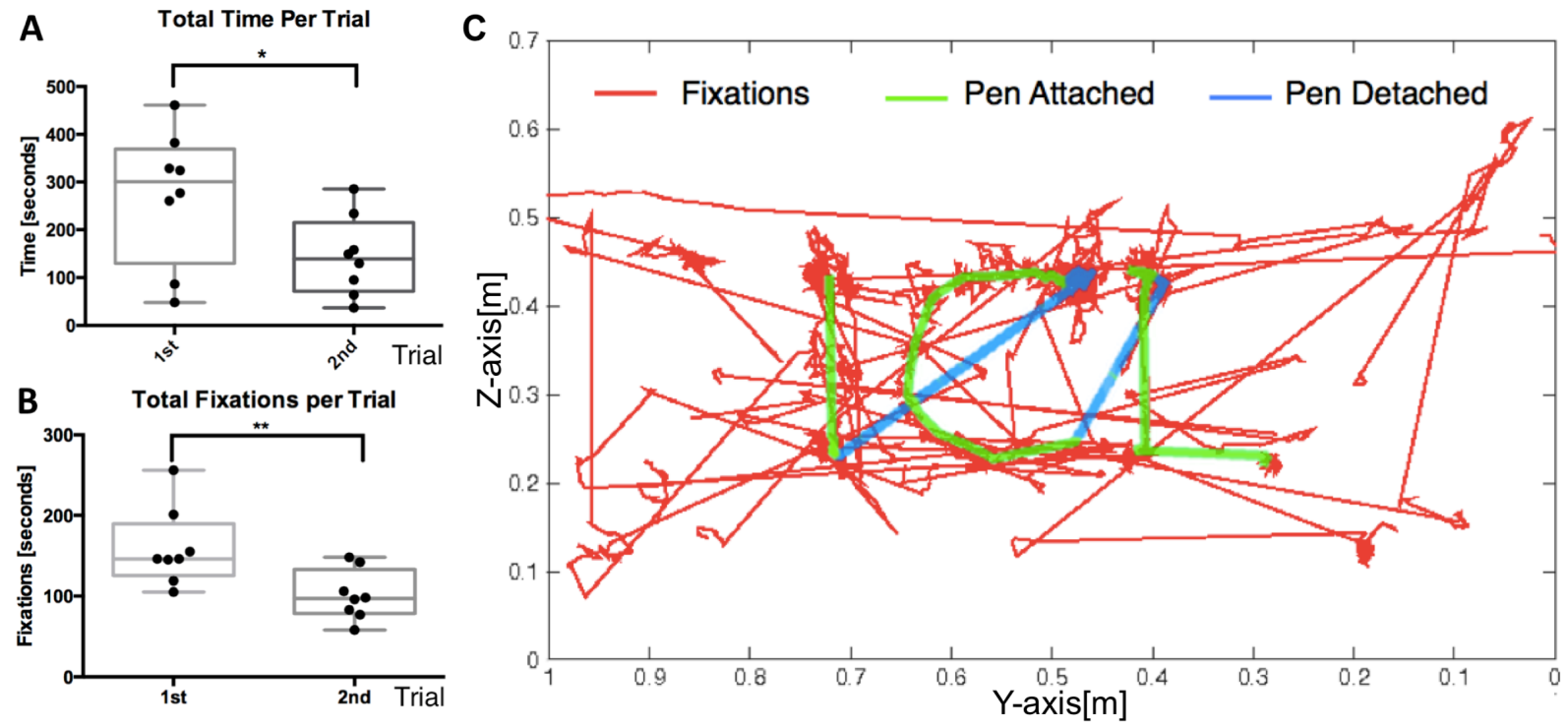

Fig. 4. (A) The total time and (B) number of selective fixations indicate the level of ability and confidence the user has in using the free-gaze control mechanism. (C) Shows the raw eye and robot movements projected onto the canvas for a user's first trial. Task-oriented and task-irrelevant eye movements are drawn as red lines, no smooth pursuit occured in this trial). Superimposed the actual robot trajectory both when the pen attached to the plane (green lines) and the pen is detached from the canvas (blue lines). This data was obtained right after calibration was completed.

as only intention commands trigger the robot to move. The figure 4 also shows that in both the total time used and the number of selective fixations which control the robot trajectory there is a significant decrease over time.

These results in Fig. 4 indicate that the users are able to learn very fast how to control the robot, the mean of the first trial with 301 seconds, this improved further in their immediately subsequent second trial to about 144 second (paired t-test on the total time $p=0.0146$ ), thus a significant decrease in the total time used between both trials. At this point the drawing time plateaued for the simple reason that we limited the robot movement speed to low joint speeds for very conservatively chosen safety reasons. The mean of the number of active fixations to move the robot was a measure of how straight-forward users controlled their system, these numbers dropped in the first trial at on average 159 to less then 101 in the second trial (this is approaches the number of straight line segments needed to render the letters smoothly). Again the number of selective fixations dropped significantly on the second trial (paired t-test, $p=0.0053$ ).

The user can close the eyes or move the head outside the head tracking box at any point of time without sending unintended commands. Studies on eye movements in natural behaviour show that fixations are more likely on objects with which a person wants to interact[6]. Therefore, fixations on a position to which the robot arm shall move to are not likely to interfere with natural gaze patterns. However, demanding the user to fixate for $600 \mathrm{~ms}$ on one point during long use might be exhausting and time consuming. A clear disadvantage of this interaction scheme is the requirement to be able to move the head. This might not be possible for people with severe paralysis, and in this case we could revert to our more advanced binocular processing approach to distinguish intentional winks (left vs right eye) from natural blinks [1].

\section{Discussion}

This paper proposes a tele-operated robotic arm - a teleprosthetic - system in which the user can direct a robot arm's movements on a flat vertical screen with their gaze, effectively writing words on the screen by a pen attached to the end point of the arm. Similarly, the user's forward and backward head movements are tracked, according to which the robotic arm is either brought in contact with the screen (signalled by a forward head motion), or pulled away from the screen (signalled by a backward head motion). A sample scenario shows that the user can effectively write the (detached) letters ICL (our institution's acronym) on a board with a remarkably short training time, i.e. literally after the first trial of first time use. Each letter is successfully drawn, and the pen is successfully detached from the board surface at the end of each letter. Since gaze saccades are highly noisy, unintentional and non-goal-directed saccades are eliminated using a simple scheme of waiting for a certain dwell time $(600 \mathrm{~ms})$ at each fixation before sending the movement command to the robot arm, thereby making sure that each saccade to control the arm are goal-directed.

Brain-Machine-Interfaces (BMIs) are the most powerful interface for paralysed and amputee users, that enable us to decode user intention and translate these into robotic actions. We demonstrated a complimentary approach based on eye tracking ${ }^{1}$. The clinical aim is always same: Extract an intention signal from a user to restore their ability to move. Conventional BMI approaches come at considerable clinical and post-clinical cost [11], while posing limitations for use in daily applications due to low information transmission bandwidths, long rehabilitation and long setup times. While 3 degree of sensing myoelectric prosthetics typically require

\footnotetext{
${ }^{1}$ Of course we can consider the eyes as a window into the brain and interpret eye movements as a high-resolution readout of brain activity, making our system a gaze-based BMI.
} 
months of intensive training, comparable comparable to training and recovery times for invasive BMI approaches for many-DoF robotic arms [12], in this paper we demonstrate that users can generate letters within minutes of first time use of our 6 DoF teleprosthetic. Powered continuous wheelchair control requires $15 \mathrm{bit} / \mathrm{s}$ and full-finger hand prosthetics 54 bit/s, well beyond the reported performance of current noninvasive BMI approaches (EEG \& EMG < 3 bit/s) [13]. The band-with limitation is mainly due to limitations set by the variability and complexity of the physiological signal noise and sensor noise [14] that are orders of magnitude higher than noise in calibrating and recording eye movementsMoreover, both invasive and non-invasive BMI approaches come at significant clinical and equipment costs. Even without taking any training/rehabilitation costs into account, these approaches can be outperformed by the efficiency and effectiveness eye tracking signals[1], [4], [3].

We demonstrate that eye tracking approach alone can be powerful the main limitation, however, is the Midas Touch problem. The complementary use of conventional BMI technology can help resolve the action selection problem, while leaving the main load of continuos end-point control to the eye tracker (and not vice versa). Extremely-invasive electrode implants to record distributed representations of intention from pre-frontal cortex brain activity in combination with eye trackers have been also proposed for Brain-MachineInterfaces [15]. Similarly eye tracking has been used to augment noisy or low-bandwidth BMI signals has been proposed before using EEG-based controlled robots [16], as well as eye-based selection from a user interface in conjunction with low data-rate signal sources for action initiation[2]. However, in contrast to previous work we use here a pure eye-movement approach to continuously control a robot end-point to perform non-trivial tasks of writing and drawing.

Even with our simple gaze-decoding decoding strategy the result allow for high user performance (despite potential confounds in decoding) and users can operate by normally looking at their task space, without requiring a distractive user interface. The fixation driven decoding of movement end-point targets works, disproving concerns that the movement of the robot arm or the pen will elicit in the user confounding smooth pursuit eye movements - in fact no smooth pursuit eye movements were detected for many of the trials observed. This enabled our users to quickly learn and operate the system within minutes of sitting down at the device for the first time in their life. Users experienced a very low cognitive load, as evidence by them being able to carry out various meaningful conversations with the experimenter during the whole writing process without detectable interruption in the control of the robot (conversation not recorded due to privacy statutes).

Our approach follows the idea of cognitive-level shared actuator control: as with our own arm, we can simply look at a target and move the arm to that target. This is in contrast to myoelectric prosthetics, where current stateof-the-art requires users to control each individual degree of freedom so that the arm eventually reaches a desired endpoint. Thus, in our gaze-based approach the joint control of the robot arm is shared between the user which specifies merely an end-point and the robot system which determines (via ROS' moveit) the low-level control.

Our free-gaze system is intuitive and quickly learned. No wearable (or implanted) devices need to be attached to the user. A paralysed wheel-chair user could drive up to the system and operate it straight away. While we tested it to date on able bodied users, effectively as a device for human robotic augmentation, our aim is to deploy this technology with paralysed or amputee users to restore their ability to move. We foresee that this type of free-gaze approach will be overcoming simultaneously several challenges in traditional BMIs by dramatically reduce cognitive load users, reducing training times, facilitate embodiment of the technology and thus boost long-term retention and patient independence.

\section{REFERENCES}

[1] W. W. Abbott and A. A. Faisal, "Ultra low-cost 3D gaze estimation: an intuitive high information throughput compliment to direct brainmachine-interfaces," J Neural Eng, vol. 9, p. 046016, 2012.

[2] E. A. Corbett, K. P. Kording, and E. J. Perreault, "Real-time evaluation of a noninvasive neuroprosthetic interface for control of reach," IEEE Trans Neural Sys \& Rehab Eng, vol. 21, no. 4, pp. 674-683, 2013.

[3] S. I. Ktena, W. Abbott, and A. A. Faisal, "A virtual reality platform for safe evaluation and training of natural gaze-based wheelchair driving," in IEEE Neural Eng (NER), vol. 7, 2015, pp. 236-239.

[4] W. W. Abbott, A. Zucconi, and A. A. Faisal, "Large-Field Study of Ultra Low-Cost, Non-Invasive Task Level BMI," in IEEE Neural Eng (NER), vol. 6, 2013, pp. 97-100.

[5] J. San Augustin, H. Skovsgaard, E. Mollenbach, M. Barret, M. Tall, D. W. Hansen, and J. P. Hansen, "Evaluation of a Low-Cost Open Source Gaze Tracker," in Eye-Tracking Research \& Applications (ETRA) Proceedings, 2009, pp. 77-80.

[6] M. Hayhoe and D. Ballard, "Eye movements in natural behavior," Trends in Cognitive Sciences, vol. 9, pp. 188-194, 2005.

[7] M. Duguleana and G. Mogan, "Using eye blinking for eog-based robot control," in Emerging Trends in Technological Innovation. Springer, 2010, pp. 343-350.

[8] R. Jacob, "The use of eye movements in human-computer interaction techniques: what you look at is what you get," ACM Trans on Information Systems (TOIS), vol. 9, no. 2, pp. 152-169, 1991.

[9] A. Y. Quigley, Morgan et Ng, "Ros: an open-source robot operating system," in ICRA workshop on open source software, vol. 3, no. 3.2, 2009 , p. 5.

[10] A. T. Duchowski, Eye Tracking Methodology: Theory and Practice. Springer, 2003.

[11] J. J. Shih, D. J. Krusienski, and J. R. Wolpaw, "Brain-computer interfaces in medicine," in Mayo Clinic Proceedings, vol. 87, no. 3. Elsevier, 2012, pp. 268-279.

[12] A. Tyler-Kabara, Elizabeth C et Schwartz, "10 degree-of-freedom neuroprosthetic control by an individual with tetraplegia," in J. Neurosurg., vol. 119, no. 2, 2013, p. A553.

[13] O. Tonet, M. Marinelli, L. Citi, P. M. Rossini, L. Rossini, G. Megali, and P. Dario, "Defining brain-machine interface applications by matching interface performance with device requirements," J Neurosci Meth, vol. 167, no. 1, pp. 91-104, 2008.

[14] A. A. Faisal, L. P. Selen, and D. M. Wolpert, "Noise in the nervous system," Nature Reviews Neuroscience, vol. 9, no. 4, pp. 292-303, 2008.

[15] M. Hauschild, G. H. Mulliken, I. Fineman, G. E. Loeb, and R. A. Andersen, "Cognitive signals for brain-machine interfaces in posterior parietal cortex include continuous $3 \mathrm{~d}$ trajectory commands," PNAS (USA), vol. 109, no. 42, pp. 17 075-17 080, 2012.

[16] L. Tonin, R. Leeb, M. Tavella, S. Perdikis, and J. d. R. Millán, "The role of shared-control in bci-based telepresence," in IEEE Systems Man Cybernetics (SMC). IEEE, 2010, pp. 1462-1466. 